\title{
CAFE: Calar Alto Fiber-fed Échelle spectrograph
}

\author{
J. Aceituno ${ }^{1}$, S. F Sánchez ${ }^{2,1}$, F. Grupp ${ }^{3}$, J. Lillo ${ }^{4}$, M. Hernán-Obispo ${ }^{5}$, D. Benitez ${ }^{1}$, L. M. Montoya ${ }^{1}$, U. Thiele ${ }^{1}$, \\ S. Pedraz ${ }^{1}$, D. Barrado ${ }^{1,4}$, S. Dreizler ${ }^{6}$, and J. Bean ${ }^{6}$ \\ ${ }^{1}$ Centro Astronómico Hispano Alemán, Calar Alto, (CSIC-MPG), C/Jesús Durbán Remón 2-2, 04004 Almería, Spain \\ e-mail: aceitun@caha.es \\ 2 Instituto de Astrofísica de Andalucía (CSIC), Camino Bajo de Huétor s/n, Aptdo. 3004, 18080 Granada, Spain \\ e-mail: sanchez@iaa.es. \\ 3 Institut fur Astronomie und Astrophysik der Universitat Munchen, Scheinerstr. 1, 81679 Munchen, Germany \\ 4 Departamento Astrofísica, Centro de Astrobiología (INTA-CSIC), ESAC campus, PO Box 78, 28691 Villanueva de la Cañada, \\ Spain \\ 5 Universidad Complutense de Madrid, Av. Complutense s/n, 28040 Madrid, Spain \\ ${ }^{6}$ University of Goettingen, Wilhelmsplatz 1, 37073 Goettingen, Germany
}

Received 10 September 2012 / Accepted 4 January 2013

\begin{abstract}
We present here CAFE, the Calar Alto Fiber-fed Échelle spectrograph, a new instrument built at the Centro Astronomico Hispano Alemán (CAHA). CAFE is a single-fiber, high-resolution $(R \sim 70000)$ spectrograph, covering the wavelength range between 3650-9800 $\AA$. It was built on the basis of the common design for Échelle spectrographs. Its main aim is to measure radial velocities of stellar objects up to $V \sim 13-14$ mag with a precision as good as a few tens of $\mathrm{m} \mathrm{s}^{-1}$. To achieve this goal the design was simplified at maximum, removing all possible movable components, the central wavelength is fixed, as is the wavelength coverage; there is no filter wheel, etc. Particular care was taken with the thermal and mechanical stability. The instrument is fully operational and publically accessible at the $2.2 \mathrm{~m}$ telescope of the Calar Alto Observatory. In this article we describe (i) the design, summarizing its manufacturing phase; (ii) characterize the main properties of the instrument; (iii) describe the reduction pipeline; and (iv) show the results from the first light and commissioning runs. The preliminar results indicate that the instrument fulfills the specifications and can achieve the planned goals. In particular, the results show that the instrument is more efficient than anticipated, reaching a signalto-noise of $\sim 20$ for a stellar object as faint as $V \sim 14.5 \mathrm{mag}$ in $\sim 2700 \mathrm{~s}$ integration time. The instrument is a wonderful machine for exoplanetary research (by studying large samples of possible systems cotaining massive planets), galactic dynamics (highly precise radial velocities in moving groups or stellar associations), or astrochemistry.
\end{abstract}

Key words. instrumentation: spectrographs - methods: observational - methods: data analysis - techniques: spectroscopic

\section{Introduction}

The Calar Alto Fiber-fed Échelle spectrograph (CAFE) is an instrument manufactured at the Centro Astronómico Hispano Alemán (CAHA) to replace FOCES (Pfeiffer 1992; Pfeiffer et al. 1998), the high-resolution Échelle spectrograph at the $2.2 \mathrm{~m}$ telescope of the observatory that was being operated during the period 1997-2010. CAFE was designed following the common concept of this type of instrument (e.g. Kaufer \& Pasquini 1998; Stahl et al. 1999; Raskin et al. 2010) and therefore its design is very similar to that of FOCES (e.g. Pfeiffer et al. 1992, $1994,1998)$. The instrument was designed to achieve a spectral resolution of $R \sim 70000$, covering the wavelength range between 3850-9800 A. The main improvements of our design were focused on increasing the stability and the sensitivity as much as possible. In particular, (i) we designed a new camera, improving the one from FOCES; (ii) we used new branch, highly efficient and long-term stable fibers; (iii) the instrument has been moved to an isolated room that is thermalized and stabilized against vibrations (as we describe below); (iv) most of the possible mobile parts in this kind of instruments were substituted by fixed elements to increase the stability of the system; and finally; (v) a new more efficient CCD with a smaller pixels was acquired.

We expected these improvements to increase the efficiency and quality of the data with respect to its predecessor.
The ultimate goal was for CAFE to achieve precisions of $\sim 10-20 \mathrm{~m} \mathrm{~s}^{-1}$ in the measurement of radial velocity of stellar objects down to $V \sim 14 \mathrm{mag}$. We were searching for the highest radial velocity accuracy for a simple and inexpensive, fast-track instrument. The goal was to produce a competitive instrument for several key areas such as exoplanetary searches, including the Kepler candidates, and the exploitation of Gaia, Perryman (2003). Thus, the $\sim 10-20 \mathrm{~m} \mathrm{~s}^{-1}$ is a compromise that allows detailed studies of large samples of moderately faint stars. However, this instrument is built for a single science case, not to provide the community of users of the observatory with a facility to cover the same studies that were performed during the past ten years with FOCES, improving the performance when feasible.

The intrument operates in a single mode (as does FEROS at the ESO 1.52 m telescope, Kaufer et al. 1997; and HARPS at the ESO 3.6 m telescope Pepe et al. 2000) and cannot be adjusted to increase either the resolution or the efficiency (in contrast to other similar instruments with more movable elements, FOCES or SOFIN at the NOT $2.6 \mathrm{~m}$ telescope, see Pronik 1995).

We describe in Sect. 2 the main properties of the instrument. The details of the design and manufacturing are given in Sect. 3 . The results from the tests performed during the first light are shown in Sect. 4.1. Finally, the tests performed during the commissioning and their results are shown in Sect. 4. A summary of the main characteristics of the instrument are included in Sect. 5. 


\section{Main properties of the instrument}

CAFE is a stationary Échelle spectrograph located at a remote laboratory in the dome building of the $2.2 \mathrm{~m}$ telescope. This room is located below the main telescope structure, separated from the rest of the building, to reduce the effects of any mechanical vibration. The mechanical stability is increased by a sophisticated pneumatic stabilization system installed in the optical bench manufactured by NewPort Corporation (model I-2000-428). This guarantees a much better stability of the instrument, increasing its performance and accuracy for radial velocity measurements of fainter objects. The room has thick reinforced concrete walls, which are part of the main support of the telescope itself. This also provides a good thermal isolation to the room. The instrument itself is separated $\sim 18 \mathrm{~m}$ from the Cassegrain focus of the telescope. The light is conducted by a single fiber and coupled to the focal plane with an improved version of the FOCES telescope module. It was manufactured by Ceramoptec and has a stainless-steel tube for it outer protection and ETFE (ethylene tetrafluoroethylene) with Kevlar for strain relief as its inner protection tube.

The optical design of the instrument camera was optimized, based on the knowledge acquired with FOCES. Based on modern optical software, a better point spread function (PSF) could be achieved over the field of view. The system has been optimized for the size of the CCD in use. Finally, we equipped the instrument with a new CCD camera, an iKon-L made by Andor Technologies, with $2048 \times 2048$ pixels of $13.5 \mathrm{mu}$. This CCD has a better quantum efficiency, lower readout-noise and higher read-out speed than the one currently used by FOCES. We expect to increase the efficiency by at least $\sim 10 \%$ with this detector.

As indicated before, the Échelle image covers the visible spectral region from 3960 to $9500 \mathrm{~nm}$, distributed in 84 orders. They are displayed in 84 spectral orders with full spectral coverage. Spectral orders are separated by 20 pixels in the blue and 10 pixels in the red. The maximum expected spectral resolution is $R=\lambda / \Delta \lambda=67000$ with a two pixel resolution element. Table 1 summarizes the main properties of the instrument and Table 2 shows their references and manufacturers.

\section{Detailed description of the instrument}

\subsection{Mechanical and thermal stability}

As indicated before, the spectrograph is located inside a controlled thermal environment to minimize any possible thermal drifts during an observing night.

The upper part of the cabinet can be lifted up with a tackle anchored on the roof of the room, as shown in Fig. 1, which provides access to the optical bench. The enclosure is kept closed during the observations.

No electronic/mechanical control system is needed once the instrument is integrated, due to its design. The only active elements inside the cabinet are the focus stage of the camera and the shutter, which are kept disconnected during a normal operation. These features add a better stability to the mechanical mounts installed on the optical bench.

Following the same philosophy, the entrance slit width of the spectrograph is fixed to $100 \mu \mathrm{m}$. The highest expected spectrograph resolution for a $2.2 \mathrm{~m}$ telescope is $R \phi=60300$ with the slit width $\phi$ entered in arcseconds; at the $2.2 \mathrm{~m}$ telescope the standard slit width corresponds to $100 \mu \mathrm{m}$, which subtends a 1.2 arcsec angle on the sky. Thus a CCD chip with $2 \times 2 \mathrm{k}$ pixels of $13.5 \mu \mathrm{m}$ distance would yield a two pixel
Table 1. Basic features of CAFE.

\begin{tabular}{ll}
\hline \hline Design & \multicolumn{1}{l}{ Échelle spectrograph } \\
Telescope & Calar Alto $2.2 \mathrm{~m}$ \\
Resolution & $62000 \pm 5000 \AA$ \\
Wavelength & $3960-9500 \AA$ \\
Sensitivity & $S N R \sim 30 \mathrm{mag} 14.5$ and $2700 \mathrm{~s}$ \\
TELESCOPE MODULE & \\
\hline Calibration lamps & Hal and ThAr \\
Entrance diaphragm & 2.4 arcsec $(200 \mu \mathrm{m})$ \\
OPTICAL FIBER & \\
\hline Type & Polymicro FBP100140170 \\
Length & $17.5 \mathrm{~m}$ \\
Inner protection tube & ETFE with Kevlar for strain relief \\
Outer protection tube & stainless-steel tube \\
Micro-lenses & N-F2, both ends \\
SPECTROGRAPH & \\
\hline Optical bench & $2400 \times 1200 \times 203 \mathrm{~mm}$ \\
Entrance slit width & $100 \mu \mathrm{m}$ \\
Grating & $31.6 \mathrm{~g} / \mathrm{mm}$ Blaze angle $=63.9$ degrees \\
Collimators & OAP1 $\lambda / 20 \mathrm{FL}=60.0 \mathrm{D}=10.0$ OAD $=7.0$ \\
& OAP2 $\lambda / 20 \mathrm{FL}=60.0 \mathrm{D}=10.0 \mathrm{OAD}=9.0$ \\
Prisms & LF5, Deviation angle 33 degrees \\
Camera & $\mathrm{f} / 3$ \\
CCD back-illuminated & $2048 \times 2048$ pixels, $13.5 \mu \mathrm{m}$ \\
\hline
\end{tabular}

Notes. (*) OAP: Off axis parabola, FL: focal length. OAD: off axis distance with respect to the center of the mirror. These magnitudes are given in inches.

Table 2. Summary of the main elements of CAFE.

\begin{tabular}{lll}
\hline \hline Item & Manufacturer & Reference \\
\hline CCD & Andor Technologies & iKON-L DZ936NBV \\
CCD cooling system & Solid State & XW-CHIL-150 \\
Pneumatic isolators & NewPort Corp & I-2000-428 \\
Collimators & NewPort Corp & OAP60-02-10Q \\
& & OAP60-04-10SQ \\
Flat mirror & Bernhard Halle Nachff & - \\
Grating & NewPort Corp & 53044ZD06-411E \\
Dispersion prisms & Prazisionsoptics Gera & 10586.09 .001 \\
Custom mounts & Indicam technologies & - \\
Fiber optics & Ceramoptec & - \\
Camera optics & Prazisionsoptics Gera & - \\
\hline
\end{tabular}

resolution of $R=72000$ with increased spectral coverage. The selection of the slit width matches the average values of seeing at the Calar Alto observatory which corresponds to 0.89 arcsec, Sánchez et al. (2007).

\subsection{Optical design}

The optical design of CAFE follows a white that of pupil concept, similar in many aspects to the one its precedent, FOCES (Baranne 1998). The optical layout is shown in Fig. 2.

The light is collected by the telescope module (see Fig. 3), which is attached to the Cassegrain focus of the telescope. It comprises halogen and thorium-argon lamps that are used for a continuum and wavelength calibration, respectively, and an optical fiber that feeds the spectrograph. A motorized device allows one to place any of the calibrations lamps in the optical path when necessary. Their beams are designed to have roughly the same $f$-ratio $(f / 8)$ as the telescope beam to illuminate the entrance aperture with the same light cone. A mirror tilted by $45^{\circ}$ 


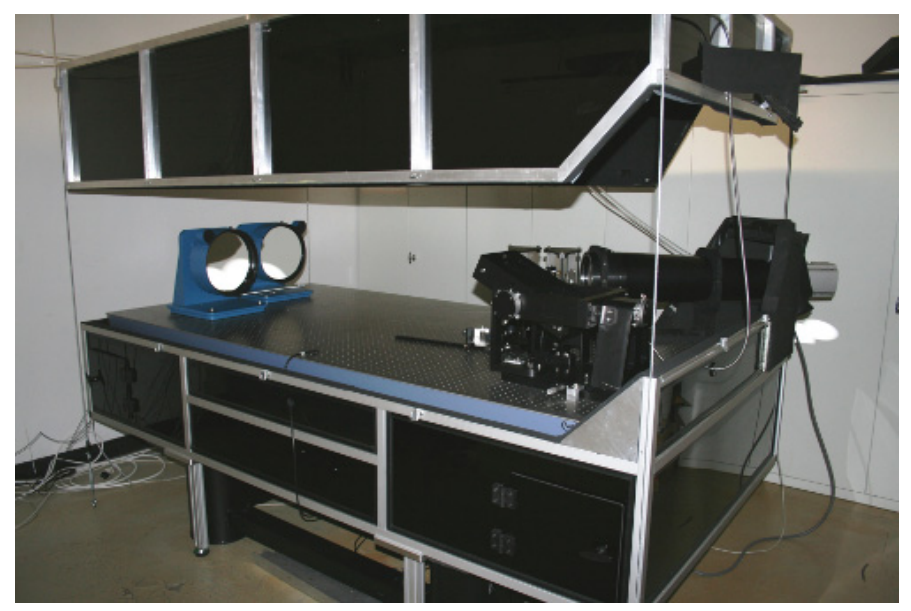

Fig. 1. CAFE spectrograph with the cabinet opened. The internal optical elements are visible on the optical bench. The upper side is as straight as a die to avoid any small apertures that can break the thermal isolation. Because of that, the upper part has to be lifted up with the help of a tackle.

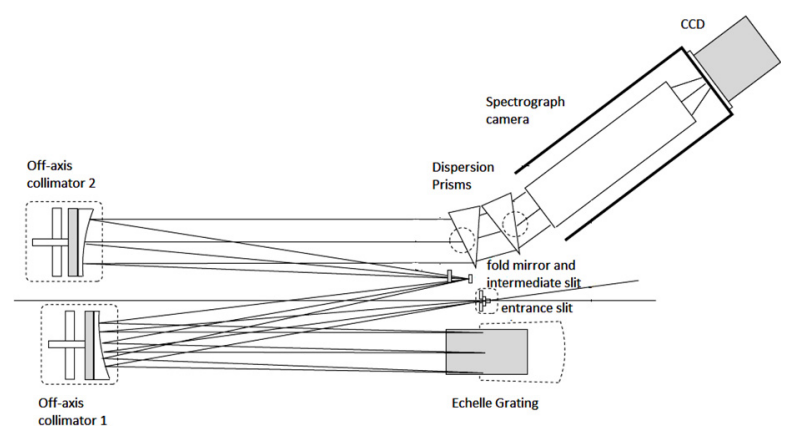

Fig. 2. Optical layout of the CAFE spectrograph with entrance slit, collimators, Échelle grating, folding mirror, prism/grism cross-disperser, and camera.

reflects the light into the fiber head and can be rotated to accept light coming from any of the comparison lamp sources. Currently halogen and thorium argon lamps are available for flat field and wavelength calibration.

Light entering into the fiber passes through a circular diaphragm that is located in a small tilted mirror just atop the fiber head. The diameter of this diaphragm is fixed to $200 \mu \mathrm{m}$ which determines the angular field accepted by the fiber and corresponds to a sky area of 2.4 arcsec. This small tilted mirror also allows guiding capabilities on the entrance aperture using the telescope guiding facilities.

Both ends of the fiber have a microlens glued to each surface. The corresponding principle is shown is Fig. 4. As for FOCES, a polymicro fiber was selected to be used with CAFE because it has been reported to have the least degradation among different available choices (Crause et al. 2008; Avila et al. 2006; Avila 1988).

A Fourier coupling is used. That means that the radial distribution of light in the telescope focal plane is transferred to an angular distribution to the fiber. Because the angular distribution of light coupled to the fiber is presevered by the fiber (modulo FDR), the Fourier lens at the end recovers the radial information from the angular one.

The first element in the optical bench is the entrance slit used to recover the original resolution when the entrance diaphragm at the Cassegrain focus is widely open to let pass starlight in

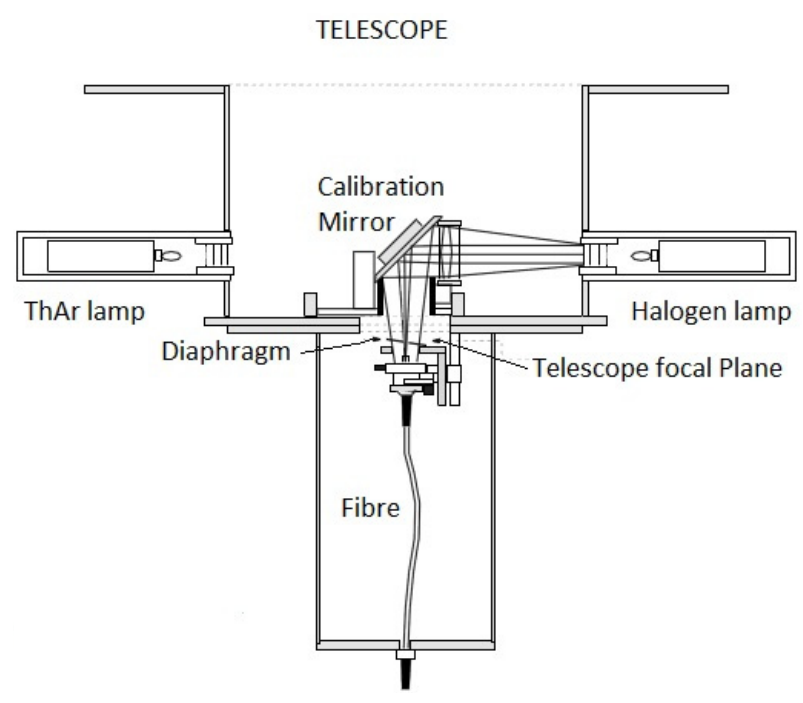

Fig. 3. Telescope module attached to the Cassegrain focus of the telescope. It contains the calibration lamps and mirror, a diaphragm, and the fiber that brings the light to the spectrograph.

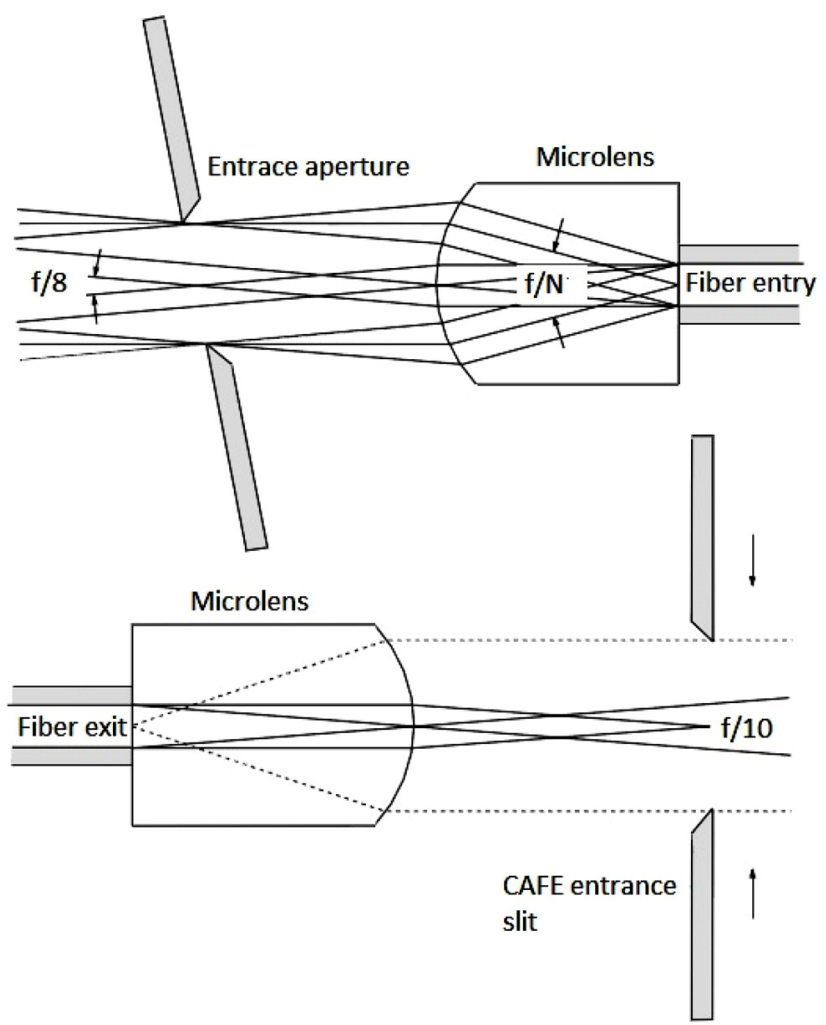

Fig. 4. Working principle of the microlenses glued to the entrance and exit of the fiber. Upper panel: fiber feed at the telescope module. Lower panel: entrance at the spectrograph (Pfeiffer et al. 1998).

case of poor seeing. It is immediately next to the folding mirror, therefore the entrance slit and its spectral image are very nearby, as close as $0.9 \mathrm{~mm}$.

To follow a white-pupil design, the spectrograph itself is collimated with two large off-axis parabolic mirrors. The $15 \mathrm{~cm}$ beam leaving the 31.6 lines/mm R2 Échelle is refocused in the vicinity of a small folding mirror, used to reflect the converging beam in the intermediate slit image, which passes a very efficient straylight baffle. 
Table 3. CAFE: List of the clearly detected orders, including the order, the wavelength range, and the spectral sampling (after re-sampling).

\begin{tabular}{|c|c|c|c|c|c|c|c|c|c|c|c|}
\hline \# & $\begin{array}{c}\lambda_{\text {start }} \\
(\AA)\end{array}$ & $\begin{array}{l}\lambda_{\text {end }} \\
(\AA)\end{array}$ & $\begin{array}{l}\Delta \lambda / \text { pix } \\
(\AA / \text { pix })\end{array}$ & \# & $\begin{array}{c}\lambda_{\text {start }} \\
(\AA)\end{array}$ & $\begin{array}{l}\lambda_{\text {end }} \\
(\AA)\end{array}$ & $\begin{array}{l}\Delta \lambda / \text { pix } \\
(\AA / \text { pix })\end{array}$ & \# & $\begin{array}{c}\lambda_{\text {start }} \\
(\AA)\end{array}$ & $\begin{array}{l}\lambda_{\text {end }} \\
(\AA)\end{array}$ & $\begin{array}{l}\Delta \lambda / \mathrm{pix} \\
(\AA / \mathrm{pix})\end{array}$ \\
\hline 60 & 9432.964 & 9558.496 & 0.0645 & 88 & 6430.853 & 6516.885 & 0.0442 & 116 & 4877.880 & 4943.502 & 0.0337 \\
\hline 61 & 9278.291 & 9401.792 & 0.0634 & 89 & 6358.568 & 6443.648 & 0.0437 & 117 & 4836.160 & 4901.236 & 0.0334 \\
\hline 62 & 9128.607 & 9250.142 & 0.0624 & 90 & 6287.889 & 6372.039 & 0.0432 & 118 & 4795.146 & 4859.686 & 0.0331 \\
\hline 63 & 8983.674 & 9103.305 & 0.0614 & 91 & 6218.764 & 6302.003 & 0.0427 & 119 & 4754.821 & 4818.833 & 328 \\
\hline 64 & 8843.271 & 8961.057 & 0.0 & 92 & 6151.141 & 6233.490 & 0.0423 & 120 & 4715.168 & 4778.662 & 326 \\
\hline 65 & 8707.188 & 8823.185 & 0.0596 & 93 & 6084.972 & 6166.449 & 0.0418 & 121 & 4676.170 & 4739.154 & 323 \\
\hline 66 & 8575.229 & 8689.491 & 0.0587 & 94 & 6020.210 & 6100.835 & 0.0414 & 122 & 4637.811 & 4700.294 & 321 \\
\hline 67 & 8447.208 & 8559.787 & 0.0578 & 95 & 5956.812 & 6036.603 & 0.0410 & 123 & 4600.075 & 4662.066 & 0.0318 \\
\hline 68 & 8322.953 & 8433.897 & 0.0570 & 96 & 5894.734 & 5973.708 & 0.0405 & 124 & 4562.947 & 4624.454 & 0.0316 \\
\hline 69 & 8202.299 & 8311.657 & 0.0561 & 97 & 5833.936 & 5912.109 & 0.0401 & 125 & 4526.413 & 4587.443 & 0.0313 \\
\hline 70 & 8085.092 & 8192.908 & 0.0554 & 98 & 5774.378 & 5851.768 & 0.0397 & 126 & 4490.459 & 4551.020 & 0.0311 \\
\hline 71 & 7971.187 & 8077.504 & 0.0546 & 99 & 5716.023 & 5792.646 & 0.0393 & 127 & 4455.070 & 4515.170 & 0.0308 \\
\hline 72 & 7860.446 & 7965.305 & 0.0538 & 100 & 5658.835 & 5734.706 & 0.0389 & 128 & 4420.234 & 4479.881 & 0.0306 \\
\hline 73 & 7752.738 & 7856.180 & 0.0531 & 101 & 5602.779 & 5677.913 & 0.0386 & 129 & 4385.938 & 4445.138 & 0.0304 \\
\hline 74 & 7647.942 & 7750.003 & 0.0524 & 102 & 5547.822 & 5622.233 & 82 & 130 & 168 & 4410.929 & 0.0301 \\
\hline 75 & 7545.939 & 7646.658 & 0.0517 & 103 & 5493.932 & 5567.634 & 0.0378 & 131 & 4318.914 & 4377.243 & 0.0299 \\
\hline 76 & 7446.621 & 7546.032 & 0.0510 & 104 & 5441.077 & 5514.086 & 0.0375 & 132 & 4286.164 & 4344.066 & 0.0297 \\
\hline 77 & 7349.883 & 7448.020 & $0 .($ & 105 & 29 & 54 & 71 & 133 & 4253.905 & 4311.388 & 295 \\
\hline 78 & 7255.624 & 7352.520 & 0.0497 & 106 & 5338.359 & 5410 & 0.0368 & 134 & 422 & 42 & 0.0293 \\
\hline 79 & 7163.752 & 7259.438 & 0.0491 & 107 & 5288.440 & 5359.444 & 0.0364 & 135 & 419 & 4247.485 & 0.0291 \\
\hline 80 & 7074.176 & 7168.682 & 0.0485 & 108 & 5239.445 & 5309.805 & 0.0361 & 136 & 4159.974 & 4216.237 & 0.0289 \\
\hline 81 & 6986.812 & 7080.168 & 0.0479 & 109 & 5191.348 & 5261.078 & 0.0358 & 137 & 4129.577 & 4185.446 & 0.0287 \\
\hline 82 & 6901.579 & 6993.811 & 0.0473 & 110 & 5144.125 & 5213.236 & 0.0355 & 138 & 4099.620 & 4155.101 & 0.0285 \\
\hline 83 & 6818.399 & 6909.536 & 0.0468 & 111 & 5097.753 & 5166.255 & 0.0352 & 139 & 4070.093 & 4125.192 & 0.0283 \\
\hline 84 & 6737.199 & 6827.267 & 0.0462 & 112 & 5052.209 & 5120.114 & 0.0348 & 140 & 4040.988 & 4095.710 & 0.0281 \\
\hline 85 & 6657.910 & 6746.933 & 0.0457 & 113 & 5007.470 & 5074.789 & 0.0345 & 141 & 4012.296 & 4066.645 & 0.0279 \\
\hline 86 & 6580.464 & 6668.467 & 0.0452 & 114 & 4963.516 & 5030.259 & 0.0342 & 142 & 3984.007 & 4037.990 & 0.0277 \\
\hline 87 & 6504.799 & 6591.805 & 0.0447 & 115 & 4920.326 & 4986.504 & 0.0340 & 143 & 3956.113 & 4009.736 & 0.0275 \\
\hline
\end{tabular}

Cross-dispersion is achieved with two LF5 prisms installed on a symmetric tandem mounting that is controlled manually. Instead of a low-order grating, a double prism for crossdispersion is used, which accounts for a more stable changing inter-order distance, and it significantly reduces local straylight in the spectrum.

Finally, the beam is imaged with an $\mathrm{f} 3$ transmission camera onto a field centered on a back-illuminated CCD with $13.5 \mu \mathrm{m}$ pixel size. The optical design of the instrument camera was optimized based on the knowledgement acquired with FOCES. Based on modern optical software, a better PSF could be achieved over the field of view. The system was optimized for the size of the CCD in use.

\subsection{Pipeline}

CAFE has been delivered with an automatic reduction pipeline. The main goal of this pipeline is to provide the user with a simple reduction of the data that can be used to test their quality during the observing run. Depending of the particularities of the science case, the data reduced with the pipeline could be used for science purposes or not, although this was not the main purpose of this package. The software is available for the community on request.

The reduction pipeline is based on R3D of Sánchez (2006). Although the package was not originally designed to reduce Échelle data, these data share some of the basic properties of any fiber-fed spectrograph. The pipeline requires four basic entries to perform the reduction: (1) the science raw-frame; (2) a bias frame acquired during the night; (3) a continuum lamp frame; and (4) an arc-lamp frame (ThAr, in this case). The main reduction steps performed are the following: (i) the raw frames are corrected by the corresponding bias; (ii) the location of the orders across the cross-dispersion axis is determined based on the intensity peak of each order at the central pixel of the CCD. Then, each order is traced along the dispersion axis reusing a similar algorithm; (iii) the science spectrum corresponding to each order is then extracted using a Gaussian extraction algorithm, see Sánchez (2006); Sánchez et al. (2012). A similar procedure is used to extract the continuum and arc lamp spectra: (iv) the continuum-extracted spectra are then used to create a master flat. To do this, a low-order polynomial function is fitted to each continuum-extracted spectrum, which is then divided by this smooth curve. The science frames are divided by this latter flat frame, correcting both the effects of the fringing and normalizing the transmission order-by-order. (v) Then, the wavelength calibration is derived for each order using the extracted arc-lamp spectra. The identified emission lines are stored in an internal look-up-table (LUT) ${ }^{1}$. Five-order polynomial function is used to derive the wavelength calibration order-by-order. (vi) The wavelength calibration is applied to the science frames, normalizing the wavelength step to the nominal one order-by-order, as shown in Table 3. (vii) Finally, a rough flux calibration is performed using a master-transmission curve derived during the commissioning runs.

\section{Commissioning}

\subsection{First light}

The first light of CAFE took place in the night of 24 May 2011, when the instrument was intalled for the first time in the telescope. The first observed science target was a bright $(V \sim 6 \mathrm{mag})$

\footnotetext{
1 A summary of this LUT is shown at http://www. caha.es/CAHA/ Instruments/CAFE/cafe/orders2.pdf
} 


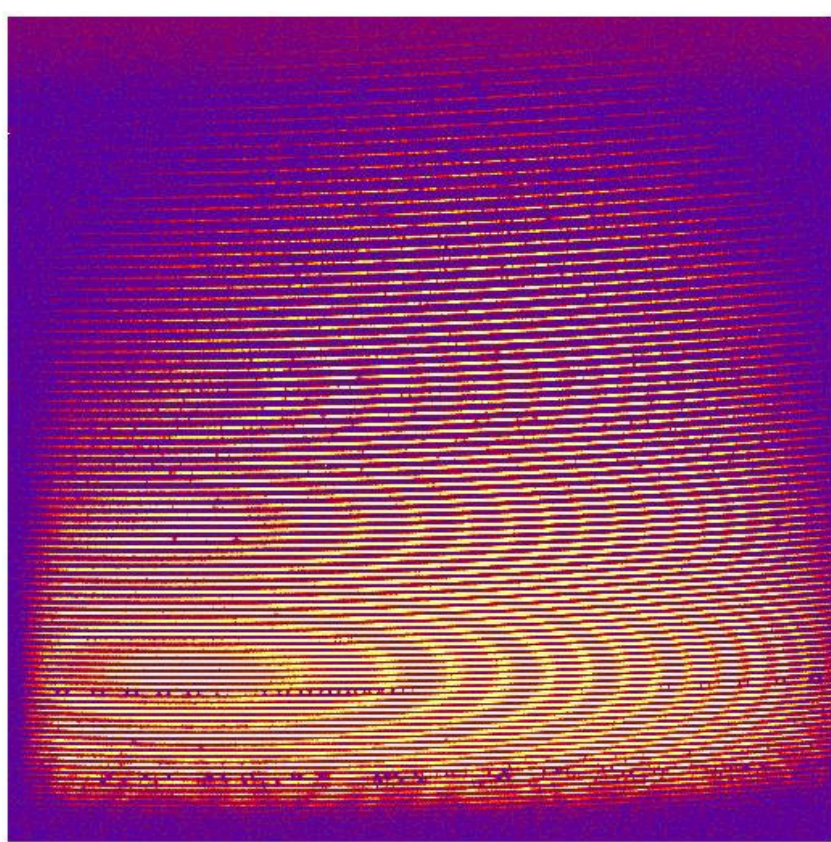

Fig. 5. Raw data of the first science frame taken with CAFE, on the star HR 4728. The orders are displayed from red at the bottom to blue at the top. For each order, the wavelength range runs from blue at the left to red at the right.
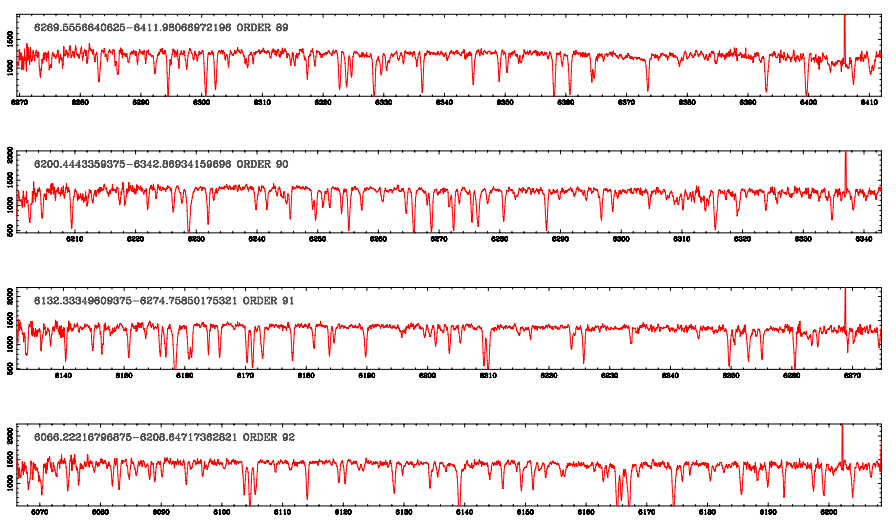

$8001.111328125-6143.53833378446$ ORDER 93

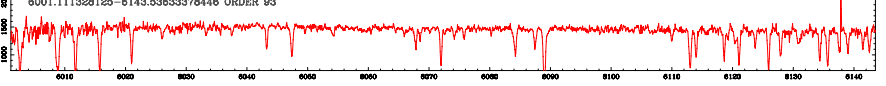

Fig. 6. Detail of a few orders of the extracted spectra of HR4728, reduced with the early version of the pipeline.

star, HR4728, selected by visibility and luminosity.The early tests performed during this night were focused on the identification of the orders and wavelength range covered by each one. The identification of the orders was a fundamental and not trivial task prior to the proper extraction and wavelength calibration of each frame.

First, the projection location of each order in the CCD, as seen in Fig. 5, is determined with the corresponding tracing routines. Figure 7 shows a vertical cut of a continuum lamp exposure used to trace these locations. The distribution of flux along the cross-dispersion axis corresponding to each of the orders is shown. For each one, the location of the peak intensity is indicated with a red (central pixel) and blue (hyperbolic centroid) cross.
Table 4. CAFE: List of detected orders.

\begin{tabular}{rc|rc|rc}
\hline \hline$\#$ & $Y$ pixel & $\#$ & $Y$ pixel & $\#$ & $Y$ pixel $^{*}$ \\
\hline 60 & 152 & 88 & 593 & 116 & 1182 \\
61 & 167 & 89 & 611 & 117 & 1207 \\
62 & 181 & 90 & 629 & 118 & 1232 \\
63 & 196 & 91 & 647 & 119 & 1258 \\
64 & 210 & 92 & 666 & 120 & 1283 \\
65 & 225 & 93 & 685 & 121 & 1310 \\
66 & 240 & 94 & 703 & 122 & 1336 \\
67 & 254 & 95 & 723 & 123 & 1363 \\
68 & 269 & 96 & 742 & 124 & 1390 \\
69 & 284 & 97 & 762 & 125 & 1417 \\
70 & 299 & 98 & 782 & 126 & 1445 \\
71 & 314 & 99 & 802 & 127 & 1473 \\
72 & 329 & 100 & 822 & 128 & 1501 \\
73 & 345 & 101 & 843 & 129 & 1530 \\
74 & 360 & 102 & 864 & 130 & 1559 \\
75 & 376 & 103 & 884 & 131 & 1589 \\
76 & 391 & 104 & 906 & 132 & 1618 \\
77 & 407 & 105 & 927 & 133 & 1649 \\
78 & 423 & 106 & 949 & 134 & 1679 \\
79 & 439 & 107 & 971 & 135 & 1710 \\
80 & 456 & 108 & 994 & 136 & 1741 \\
81 & 472 & 109 & 1016 & 137 & 1773 \\
82 & 489 & 110 & 1039 & 138 & 1805 \\
83 & 506 & 111 & 1062 & 139 & 1837 \\
84 & 523 & 112 & 1086 & 140 & 1870 \\
85 & 540 & 113 & 1109 & 141 & 1905 \\
86 & 557 & 114 & 1133 & 142 & 1938 \\
87 & 575 & 115 & 1158 & 143 & 1972 \\
\hline & & & & &
\end{tabular}

Notes. ${ }^{(*)}$ For each order we show the $y$-axis pixel at the $x$-axis center pixel in the CCD. The data shown here were obtained during the commissioning. An updated table can be found at http://w3.caha.es/ CAHA/Instruments/CAFE/index.html

We identified the orders after tracing and extracting a ThAr frame (following the reduction steps explained in Sect. 3.3).

Table 4 shows the 84 detected orders, including the $Y$-coordinate of the peak intensity of each order projected in the $\mathrm{CCD}$ at the central pixel in the $X$-axis, i.e., the pixel marked with a red cross in Fig. 7. Table 3 shows the wavelength range sampling for each order, after normalizing to a common spectral sampling per pixel.

\subsection{Efficiency of the instrument}

The net efficiency of the instrument was derived by comparing the expected photons obtained from a certain source with the real number of photons acquired by the instrument. To do this, we used the exposures on the calibration stars during the night of 17 July 2011. We observed five different calibration stars during that night. The grammes were reduced with the pipeline (described bellow), and finally we extracted a (flux)-uncalibrated spectra for each of the orders, in counts. Then, we transformed counts into photoelectrons using the gain of the CCD for each wavelength $\left(n_{\mathrm{e}, \mathrm{det}}\right)$.

To derive the number of expected photoelectrons one needs to use the known flux-calibrated spectra of the considered standard star. The observed spectrophotometric standard stars were all extracted from the Oke (1990) catalog ${ }^{2}$, whose flux density is provided in units of $10^{-16} \mathrm{erg} \mathrm{s}^{-1} \AA^{-1} \mathrm{~cm}^{-2}$. The amount of flux $(F)$ at a certain wavelength $\lambda$ in a wavelength interval $\Delta \lambda$

2 http://www.caha.es/pedraz/SSS/Oke/oke.html 

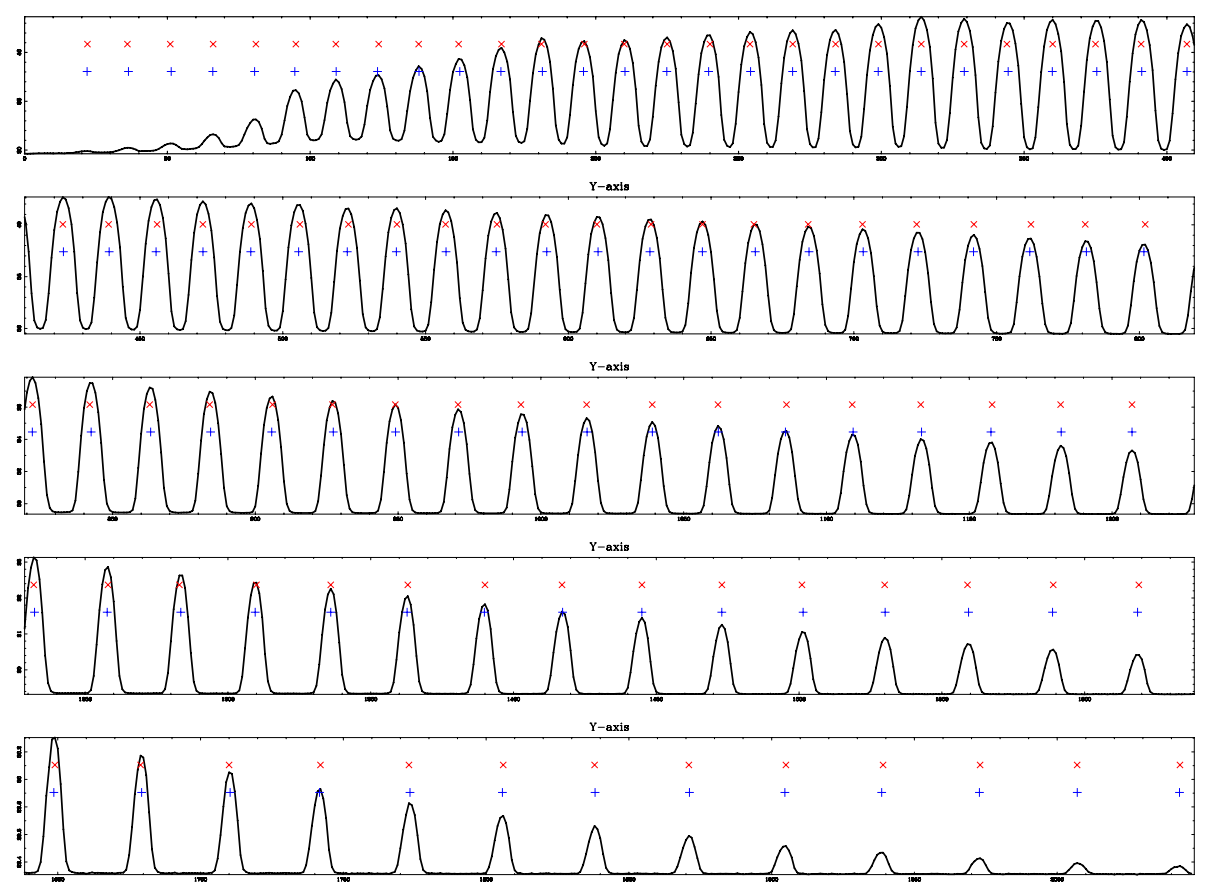

Fig. 7. Vertical section of a continuum exposure at the central $X$-axis pixel, showing the projection on the CCD of each order, from red wavelengths (top-left) to blue ones (bottom-right). The location of the peak intensity of each order is marked with a red (central pixel) and a blue cross (centroid).

of a star (or any other target), with flux density $f_{\lambda}$ collected by a telescope of collecting area $(\Delta S)$ in a time interval $(\Delta t)$ is given by the formula

$F=f_{\lambda} \cdot \Delta S \cdot \Delta t \cdot \Delta \lambda$

On the other hand, the energy of a single photon is

$f_{\text {photon }}=\frac{h c}{\lambda}$.

The ratio between both quantities gives the number of expected photoelectrons $\left(n_{\mathrm{e}, \exp }\right)$. This number can be directly compared with the number of detected photoelectrons, obtained from the reduced data as described above. Finally, the net efficiency of the instrument, as a function of the wavelength, is defined as

efficiency $=\frac{n_{\mathrm{e}, \mathrm{det}}}{n_{\mathrm{e}, \mathrm{exp}}}$.

Figure 8 shows the derived efficiency of the instrument (plus telescope and detector), as a function of the wavelength. CAFE is significantly more efficient than FOCES at any wavelength range Pfeiffer et al. (1998). In comparison with other similar Échelle spectrographs, available at telescopes of a similar aperture, CAFE has a similar peak efficiency as FEROS (Guenther et al. 1999; Kaufer et al. 1999), although this instrument is more efficient in the blue end. The main difference seems to be the efficiency of the CCD, which for FEROS has a coating whose efficiency changes from blue to red from one side to another across the CCD (i.e., its coating is optimized for the wavelength range covered by each order). Therefore, a simple way to improve the efficiency of the instrument even more would be to acquire a similar CCD, although this is not considered in the near future.

\subsection{Instrumental focus and wavelength resolution}

One of the main goals in designing and manufacturing CAFE was to achieve a resolution better than that of its predecessor,

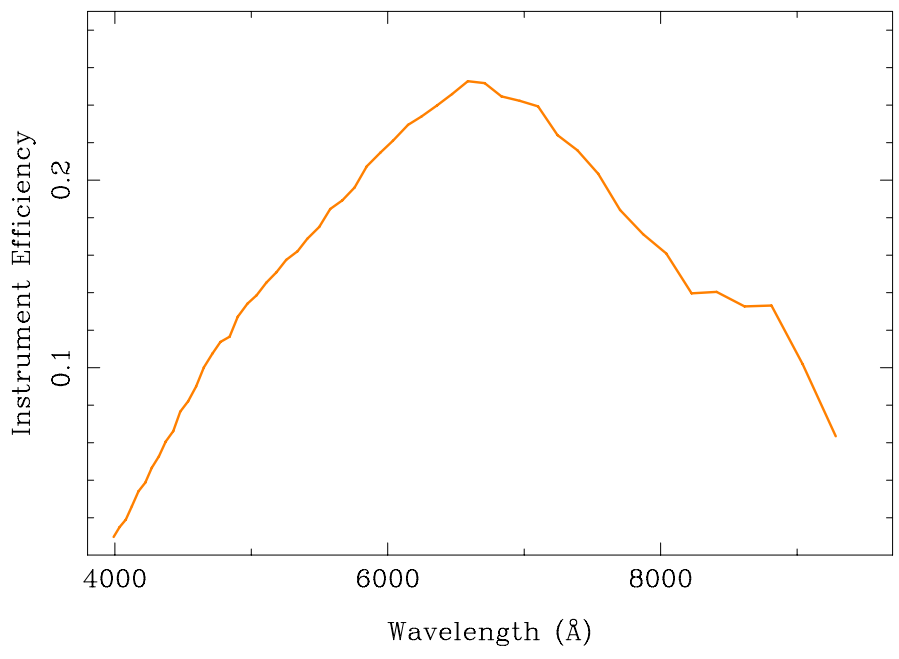

Fig. 8. Overall CAFE efficiency (including telescope) derived from the analysis of the calibration star taken during the commissioning run.

with a better efficiency and stability. CAFE was designed to achieve a maximum resolution of $R \sim 70000$ (Sánchez et al. 2007) in ideal conditions.

The instrument resolution is defined as the ratio between the wavelength $\lambda$ and the minimum range of wavelengths that can be resolved $\Delta \lambda$. In practice, $\Delta \lambda$ is derived from the FWHM in the spectral direction of the emission lines of arc-lamps. Assuming that these lines are (in general) unresolved, the FWHM measures the instrumental resolution, i.e., the minimum wavelength elements to be resolved. Early measurements in the laboratory indicate that the FWHM of these emission lines were around $\sim 2.2$ pixels, which mostly corresponds to a resolution $R \sim 67000$ at the average wavelength sampled by the instrument $(\lambda 6500 \AA)$.

The spectral resolution can be derived in detail after a proper identification of the orders, the wavelength range covered by 

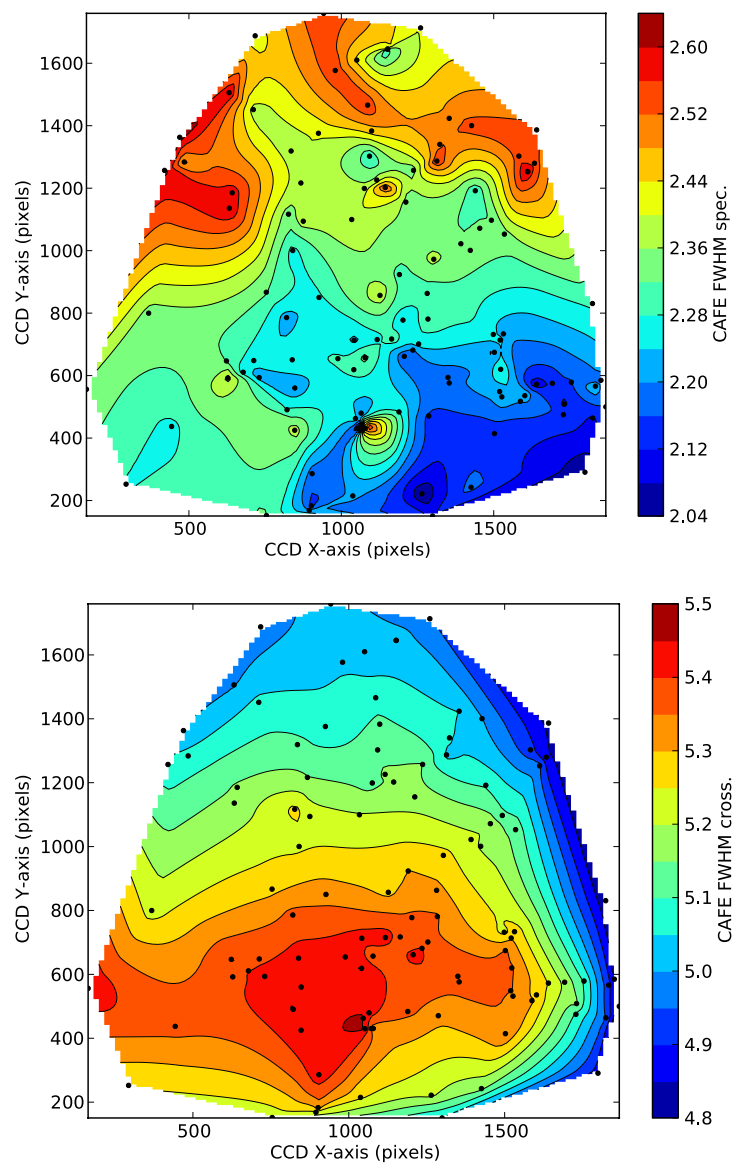

Fig. 9. CAFE: FWHM of the arc-lamp emission lines. The upper panel shows the distribution of the FWHM of the arc-lamp emission lines along the dispersion axis ( $X$-axis), across the field-of-view of the CCD. The lower panel shows a similar distribution for the FWHM along the cross-dispersion axis.

each of them, and the corresponding sampling ratio per pixel. Once an arc-lamp exposure is reduced with the pipeline described below, each of the 336 identified arc emission lines are fitted with a one-dimensional Gaussian function in both the dispersion and cross-dispersion directions, deriving the FWHM in both axis. The FWHM in the cross-dispersion axis illustrates how well each order is separated form the adjacent ones, and by which amount they are contaminated by cross-talk. Figure 9 shows the distribution of both FWHMs across the field-of-view of the CCD.

On the other hand, the FWHM in the dispersion axis is a direct measurement of the spectral resolution. Once it is derived the FWHM of each of the identified lines, a clipping algorithm rejects the values $(<10 \%)$ that deviated more than $3 \sigma$ of the mean one. The derived FWHM is multiplied by the step in wavelength per pixel and divided into the wavelength of the line to derive the instrumental resolution $(R)$.

Figure 10 shows the distribution of the spectral resolution along the wavelength derived from the first ThAr lamp observed in the night of 16 June 2011. Similar distributions are found for any arc calibration frames taken during the commissioning run. On average, the instrumental resolution estimated on real data corresponds to $\sim 63000 \pm 4000$. There is a clear trend in the resolution from the blue to the red range, with the resolution being $\sim 60000$ in the blue end $(\sim 4000 \AA)$, and about $\sim 70000$ in the red end $(\sim 9500 \AA)$. This median value is statistically dominated

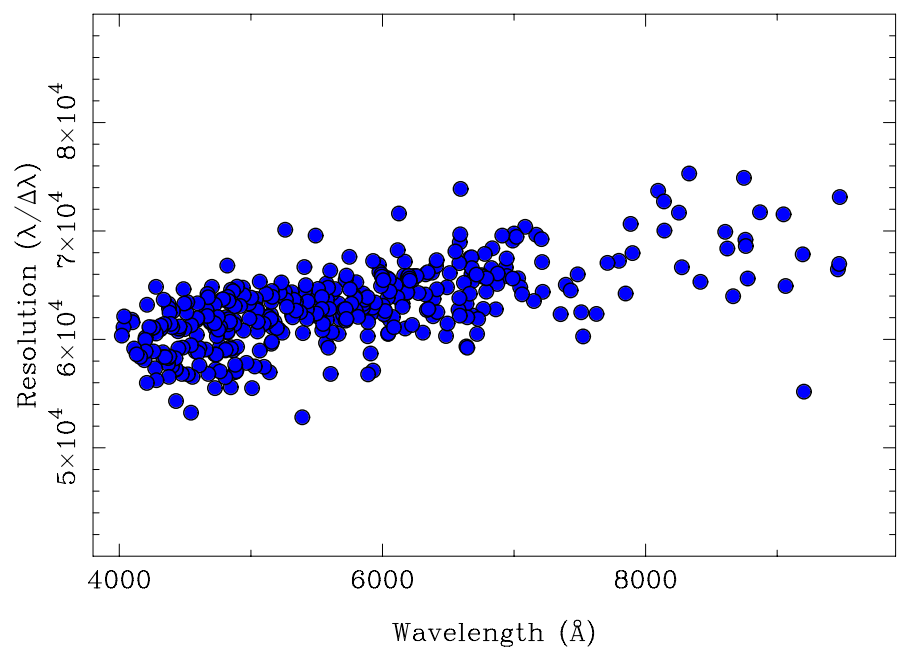

Fig. 10. CAFE: wavelength resolution derived from the estimation of the FWHM of the identified ARC emission lines in the dispersion axis.

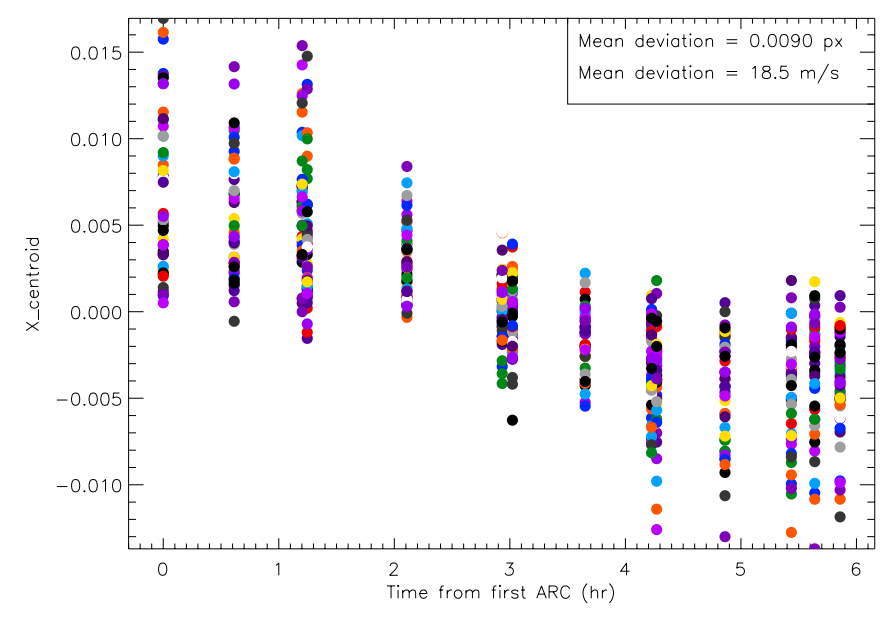

Fig. 11. Stability of CAFE along a six-hours observing night on June 23, 2012. The mean dispersion of the centroid position in the $X$-axis for the different Th-Ar frames is shown. Forty-three spots, represented with different colors, were used in $14 \mathrm{ThAr}$ arc images to generate this figure.

by the values at wavelengths bluer than $\sim 5500 \AA$, where there are more identified arc lines. As anticipated, the resolution at the average wavelength of $\sim 6500 \AA$ is $\sim 65000$.

Following the specifications of the design, CAFE was built and calibrated to produce a similarly accurate image quality at any position across the CCD. That is, it was a goal of the design to have the same FWHM in the ThAr spots from blue to red arm at any order, at least on the spectral axis. A constant FWHM (in pixels) produces an unavoidable change in the resolution along the spectral range, as the one observed here.

This resolution is, on average, higher than the one that could be achieved by FOCES ${ }^{3}$. With that instrument, it was possible to reach a resolution of about $\sim 65000$ only when observing with the narrowest slit width (with the consequent loss of signal-tonoise), and it was not possible to achieve a resolution higher than this value.

http//www.caha.es/pedraz/Foces/spec_resol.html 

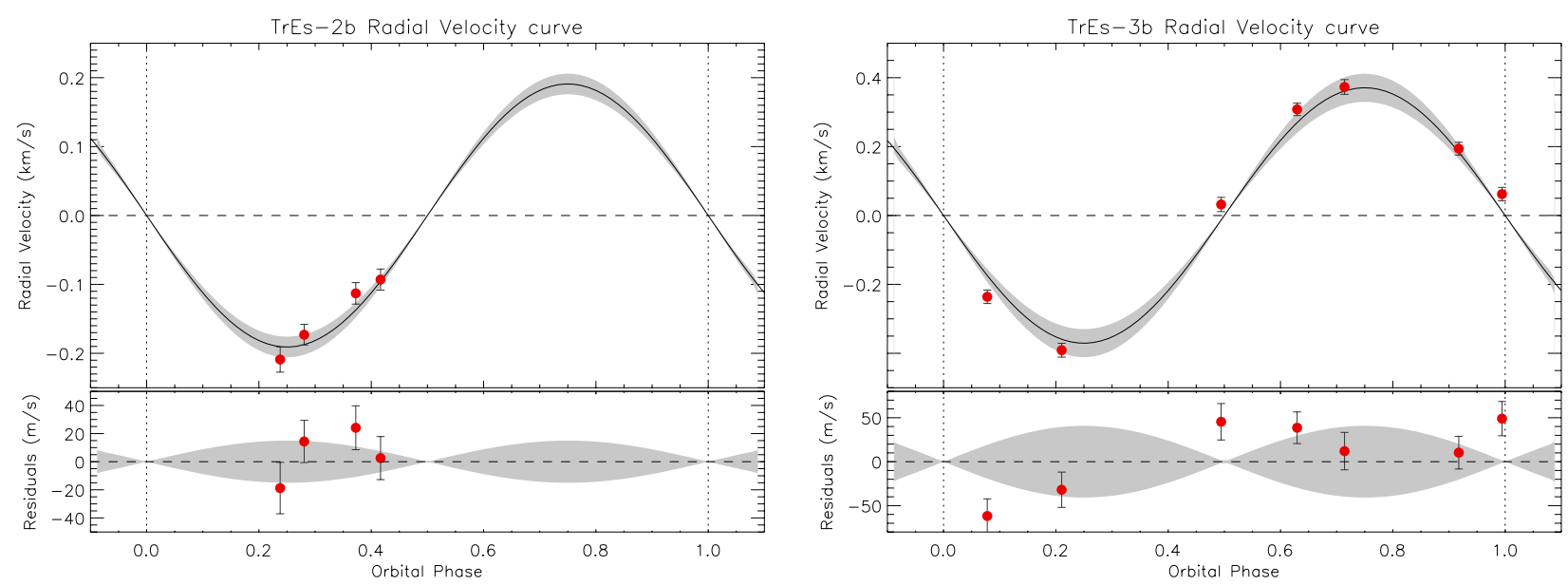

Fig. 12. Radial velocity curves for two well-known extra solar planets, TrEs-2b (left panel), and TrEs-3b (right panel), derived from the early measurements taken during the CAFE commissioning run. Red circles represent the derived values for the radial velocity. The black solid line represents the theoretical curve assuming the simple expression $v_{r}=\frac{2 \pi a}{P} \sin (i) \frac{M_{\mathrm{p}}}{M_{\mathrm{p}}+M_{\mathrm{s}}} \sin (\phi)$, where a is the semi-major axis, $P$ is the orbital period, $i$ is the orbital inclination, and $\phi$ is the orbital phase. The shaded region was calculated by error propagation of the published values in the previous expression. The lower panel shows the residuals for the fit.

\subsection{Stability of the focus}

CAFE was designed to stabilize the camera focus (and therefore the resolution) as much as possible. Consequently, compared with its predecesor, many moving elements were replaced by fixed ones. To test if the goal was achieved, one needs to repeat the measurements described in Sect. 4.3 for different ARC-lamp exposures taken under different conditions.

So far, we have obtained $34 \mathrm{ThAr}$ ARC lamp frames from the commissioning run. We repeated the procedure described before for each of them, deriving the mean (and standard deviation) of the FWHMs values measured for each of them. Figure 13 shows the distribution of these mean FWHMs along the time (upper panel) and along the internal temperature of the instrument (lower panel). It is important to note here that although the instrument is equipped with a thermal controlling system, this system was not operational during the commissioning run. Therefore, any effect of the temperature on the focus (and the stability of the resolution) should be detected in this plot.

The average value of the FWHMs along the dispersion axis range yields between $2.3-2.35$ pixels, without any significant variation with the time and/or temperature. The variation across the field is much larger (see Fig. 9) than any possible detected variation along the time/temperature. In this regard, the goals of the design have been completely fullfilled.

It is required to (1) perform this analysis for any of the observed nights hereafter to feed the statistics with more data, and (2) repeat the analysis if the focus is reset.

\subsection{Signal-to-noise}

CAFE was designed to be more efficient than FOCES. To achieve this, new branch fibers, optics, higher-efficiency elements and less movable elements were included.

To determine if we achieved this goal, we used the fluxcalibrated spectra of the different objects observed during the commissioning run to derive the average signal-to-noise ratio $(\mathrm{S} / \mathrm{N})$, per spectral pixel. To do this, an automatic procedure was included in the CAFE pipeline that compares the extracted signal with the derived variance after propagating the different reduction steps.
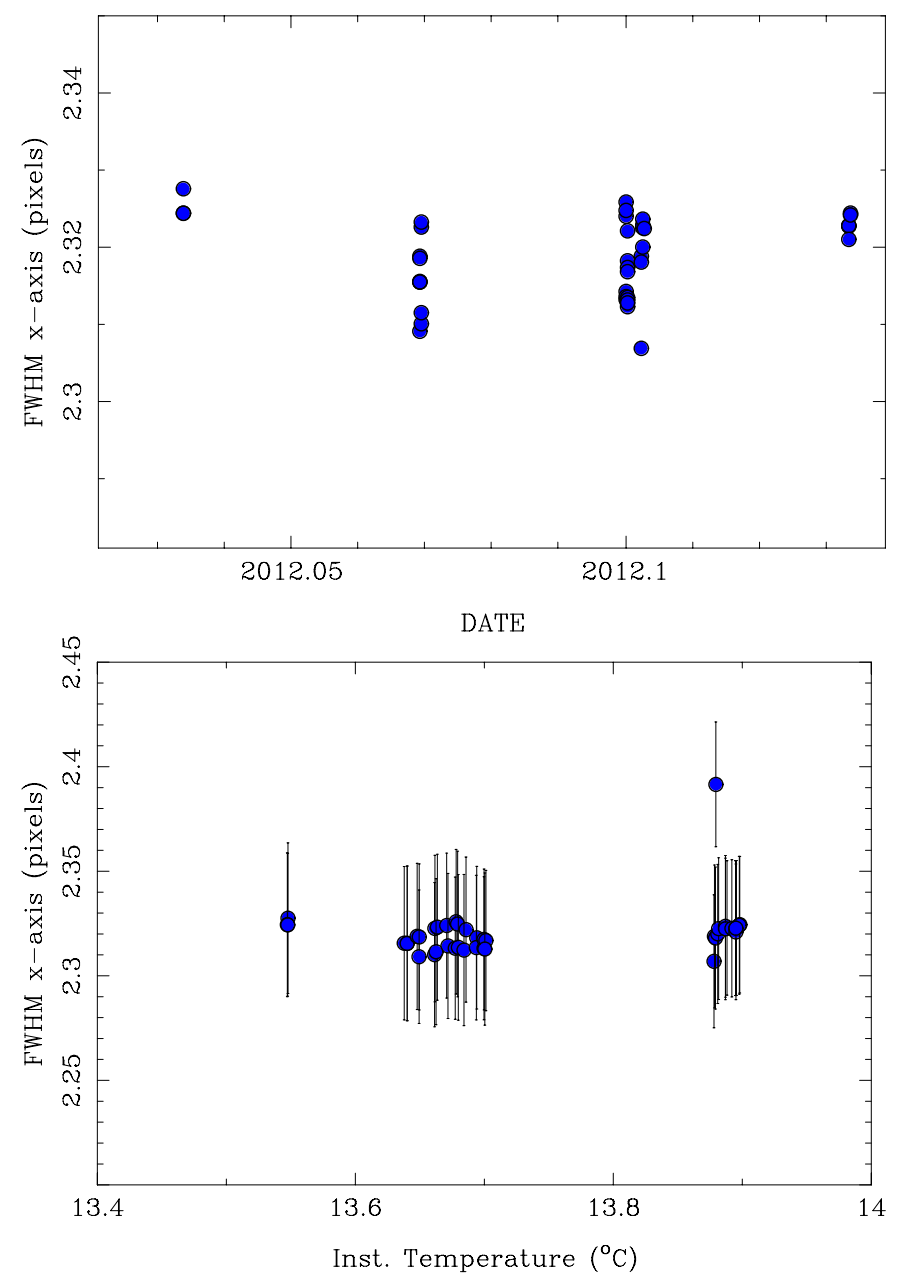

Fig. 13. CAFE: stability of the spectral resolution. The upper panel shows the distribution of the average FWHM of the ARC emission lines along the dispersion axis (e.g., the one shown in Fig. 10) for the different ARC frames taken during the commissioning run. The lower panel shows a similar distribution along the internal temperature of the instrument, measured with the four sensors described in Sect. 3.1.

Figure 14 shows the theoretical curves derived from an exposure-time calculator for CAFE. The achieved $\mathrm{S} / \mathrm{N}$ as a 
Table 5. Summary of the observed objects along the commissioning nights.

\begin{tabular}{|c|c|c|c|c|}
\hline Date & $\begin{array}{l}\text { Object } \\
\text { Name }\end{array}$ & $\begin{array}{r}\text { Exposure } \\
\text { Time }\end{array}$ & $\begin{array}{r}\mathrm{AB} \\
(\mathrm{mag})\end{array}$ & $S / N$ \\
\hline $11-06-15$ 22:30:10 & HAT-P-12b & 2700 & 12.4 & 57.3 \\
\hline $11-06-15$ 23:22:36 & WASP-24b & 1800 & 11.3 & 67.7 \\
\hline $11-06-1600: 29: 44$ & KOI-561B & 3600 & 13.6 & 45.3 \\
\hline 11-06-16 00:45:04 & HD 154345 & 30 & 6.8 & 56.3 \\
\hline $11-06-1600: 49: 41$ & HD 154345 & 60 & 6.3 & 48.3 \\
\hline $11-06-1601: 27: 21$ & TrES-2b & 1800 & 11.5 & 75.4 \\
\hline $11-06-16$ 02:24:05 & Tres-3b & 2700 & 12.2 & 70.1 \\
\hline $11-06-16$ 02:57:13 & TrES-2b & 1800 & 11.3 & 75.9 \\
\hline 11-06-16 03:03:00 & THD 182572 & 30 & 4.7 & 62.5 \\
\hline $11-06-17$ 21:13:45 & HZ44 & 1800 & 11.8 & 53.0 \\
\hline 11-06-17 21:46:51 & Feige66 & 1200 & 10.8 & 62.5 \\
\hline 11-06-17 22:14:10 & $B D+33 d 2642$ & 1200 & 11.4 & 56.2 \\
\hline $11-06-17$ 23:14:34 & TrES-3b & 2700 & 12.6 & 46.7 \\
\hline $11-06-17$ 23:19:53 & HD 115404 & 30 & 6.7 & 53.4 \\
\hline $11-06-17$ 23:35:51 & HD 139323 & 30 & 7.20 & 35.1 \\
\hline 11-06-18 00:11:11 & P330D & 1800 & 12.9 & 37.7 \\
\hline 11-06-18 00:52:22 & TrES-2b & 1800 & 11.4 & 42.7 \\
\hline 11-06-18 01:04:29 & HD 151541 & 50 & 7.2 & 45.8 \\
\hline 11-06-18 01:33:24 & $B D+25 d 4655$ & 600 & 10.6 & 34.1 \\
\hline 11-06-18 02:32:04 & TrES-3b & 2700 & 12.2 & 57.5 \\
\hline 11-06-18 03:12:31 & TrES-2b & 1800 & 11.0 & 42.5 \\
\hline $11-06-18$ 03:23:20 & HD 090404 & 50 & 7.1 & 64.6 \\
\hline 11-06-18 20:58:34 & P330D & 1800 & 13.2 & 33.4 \\
\hline 11-06-18 21:04:44 & HD 115404 & 30 & 7.1 & 31.3 \\
\hline 11-06-18 22:03:56 & TrES-3b & 2700 & 12.3 & 58.2 \\
\hline 11-06-18 23:00:47 & Kepler-561 & 2700 & 13.8 & 30.0 \\
\hline $11-06-18$ 23:35:28 & TrES-2b & 1800 & 11.3 & 61.4 \\
\hline 11-06-18 23:44:03 & HD 139323 & 30 & 7.3 & 46.7 \\
\hline 11-06-19 00:41:05 & TrES-3b & 2700 & 12.0 & 58.5 \\
\hline 11-06-19 01:30:06 & Kepler-561 & 2700 & 13.6 & 32.3 \\
\hline 11-06-19 02:08:19 & TrES-2b & 1800 & 11.0 & 61.2 \\
\hline 11-06-19 02:18:33 & HD 151541 & 50 & 7.1 & 52.3 \\
\hline 11-06-19 03:12:25 & Kepler-561 & 2700 & 14.5 & 20.0 \\
\hline 11-06-19 03:25:07 & HD 190404 & 50 & 6.8 & 62.1 \\
\hline
\end{tabular}

function of time for a given magnitude has been included for the corresponding theoretical curve.

Table 5 shows the results of this $\mathrm{S} / \mathrm{N}$ analysis, for the targets observed during the commissioning run, including the date, the name of the target, the $V$-band magnitude and the $\mathrm{S} / \mathrm{N}$ at the average wavelength of this band ( $5500 \AA)$. However, a similar $\mathrm{S} / \mathrm{N}$ within $20 \%$ is derived for the full wavelenght range between 5000-6500 A, depending more on the shape of the continuum of the considered target (e.g. stellar type) than on the properties of the instrument. These results can be directly compared with those derived for FOCES ${ }^{4}$. We highlight here the results derived for the G5 star with $V$-band magnitude of 6.9 mag, derived with FOCES, from which we obtain a $S / N \sim 41$ for an exposure time of $60 \mathrm{~s}$. This can be compared with the result we obtain with CAFE for HF151541, a $V \sim 7.1 \mathrm{mag}$ star, for which we obtain an $S / N \sim 68$ with a exposure time of $60 \mathrm{~s}$. The faintest object listed in the FOCES reference web-page is a 0p star with a luminosity of $V \sim 10.5 \mathrm{mag}$, for which we obtained a spectra with an $S / N \sim 25$ with an exposure time of 600 s. A similar star, $\mathrm{BD}+25 \mathrm{~d} 4655, V \sim 10.6 \mathrm{mag}$, was observed with CAFE, for which we obtained a spectrum with a $S / N \sim 45$, with a similar exposure time $\left(t_{\exp } \sim 600 \mathrm{~s}\right)$.

\footnotetext{
4 http://www.caha.es/pedraz/Foces/signal.html
}
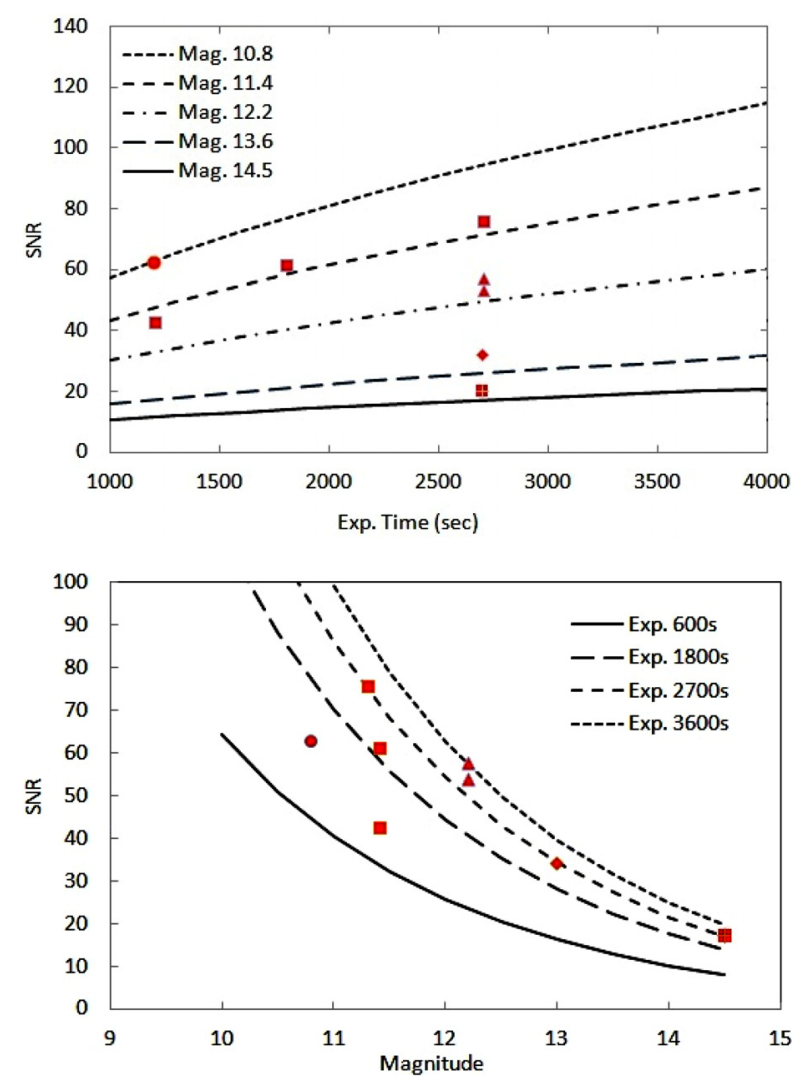

Fig. 14. Upper panel: the $\mathrm{S} / \mathrm{N}$ as a function of time for a given magnitude. Some experimental data for the corresponding magnitude are plotted on the theoretical curves for which magnitudes were derived. Lower panel: the $\mathrm{S} / \mathrm{N}$ is shown as a fucntion of magnitude for different fixed exposure times. The same experimental data as in the upper case were plotted to be consistent.

In average, the $\mathrm{S} / \mathrm{N}$ derived by CAFE is twice larger than the one derived with FOCES for targets with similar luminosity and using similar exposure times.

Figure 15 summarizes the results of this analysis, showing in two different representations the dependency of the $\mathrm{S} / \mathrm{N}$ on the intrinsic brightness of the observed object and the adopted exposure time. Based on these results, the limiting magnitude of CAFE would be $\sim 15$ mag for an exposure time of one hour with an $\mathrm{S} / \mathrm{N}$ of $\sim 20$. We consider the goal of providing an instrument more efficient than FOCES to be met.

These figures/tables should be extended with any additional information provided from any further observing run to derive much more accurate results/expectations.

\subsection{Radial velocity measurements}

Figure 11 shows the high stability of CAFE along a six hour observing night on June 23, 2012. We used 43 spots in $14 \mathrm{ThAr}$ arc images during the night to follow their centroid values (in the $X$ and $Y$ directions). The mean dispersion of the centroid position in the $X$-axis for the different images is 0.009 pixels while 0.010 pixels is found for the $Y$ direction, resulting in a radial velocity precision of around $18.5 \mathrm{~m} / \mathrm{s}$ and $21.2 \mathrm{~m} / \mathrm{s}$, respectively. It is also important to note the slight dependence on the centroid position during the night. However, this can be easily corrected by using the closest ThAr arc to wavelength-calibrate the science images and hence achieve the mentioned precisions. We detect different trends depending on each particular night; 

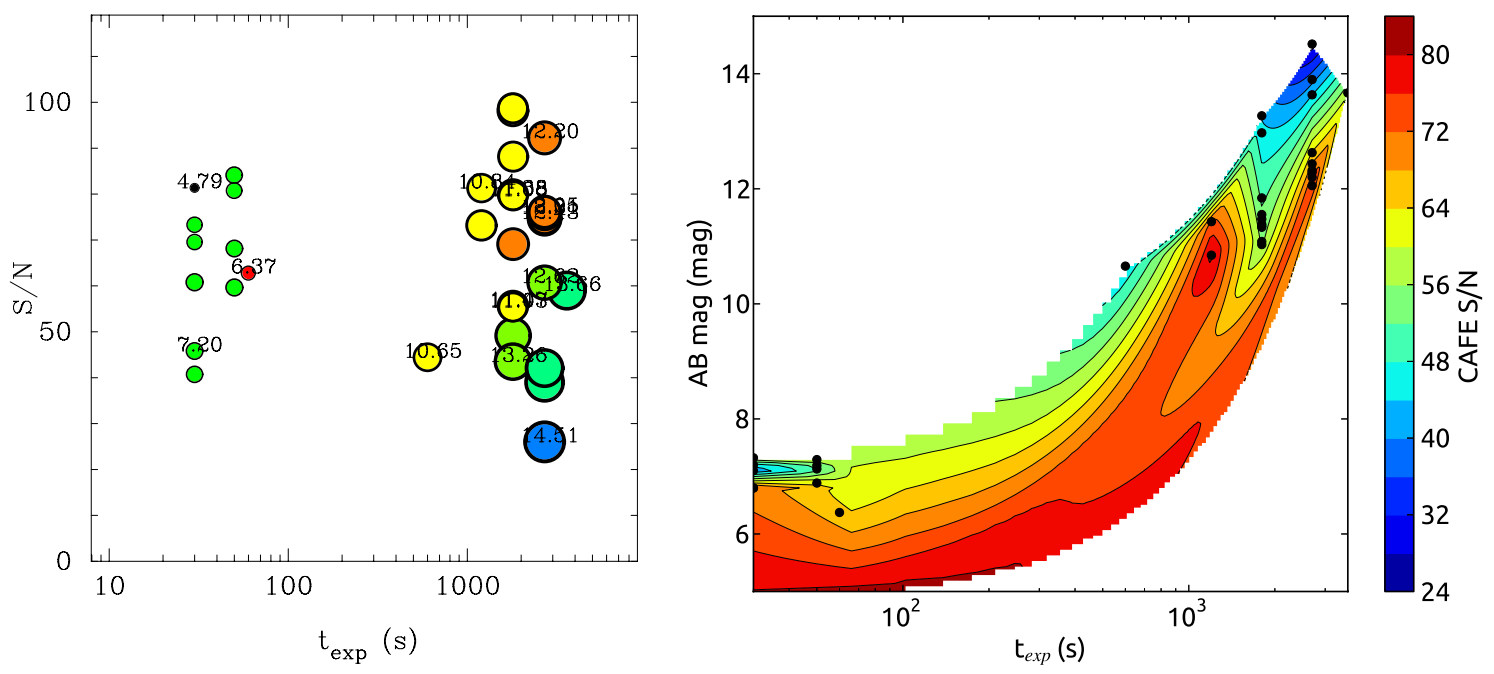

Fig. 15. CAFE: results from the $\mathrm{S} / \mathrm{N}$ analysis. The left panel shows the distribution of the $\mathrm{S} / \mathrm{N}$ during the exposure time for the different observed objects. The color/size of the plotted symbols indicates the brightness of the object. The right panel shows the $\mathrm{S} / \mathrm{N}$ distribution as a function of the brightness and the exposure time.
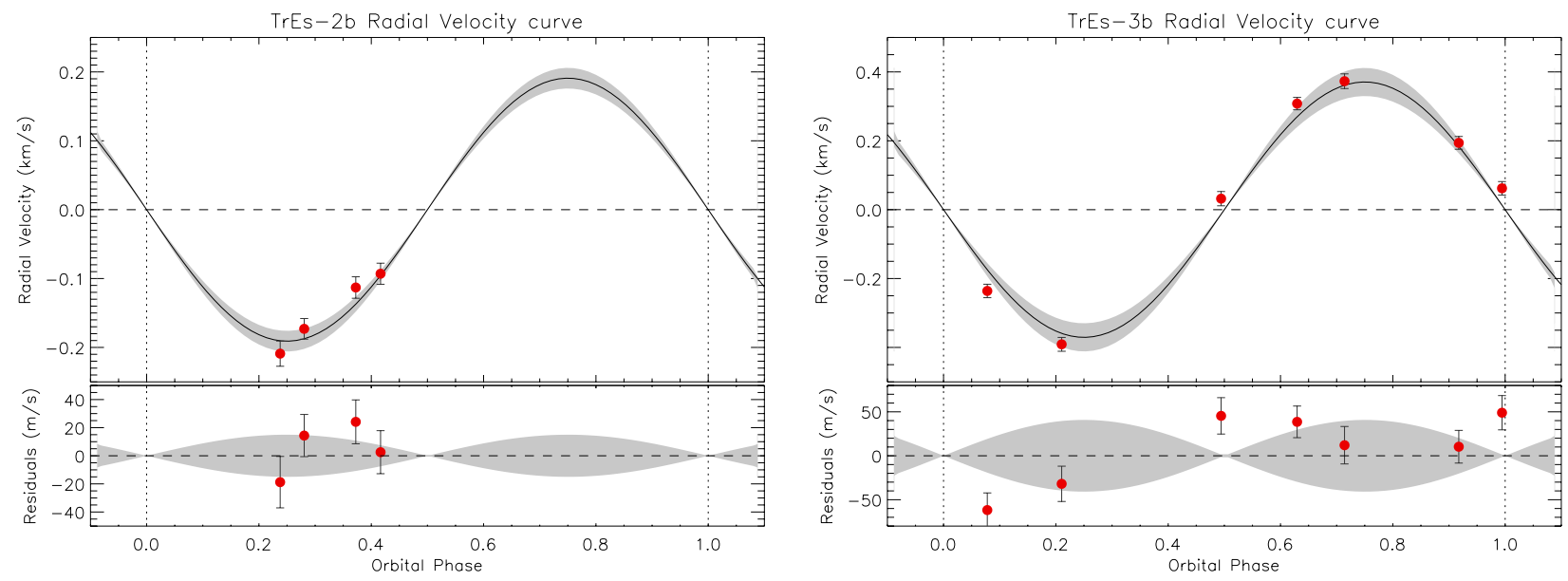

Fig. 16. Radial velocity curves for two well-known extra-solar planets, TrEs-2b (left panel), and TrEs-3b (right panel), derived from the early measurements taken during the CAFE commissioning run. Red circles represent the derived values for the radial velocity. The black solid line represents the theoretical curve assuming the simple expression $v_{r}=\frac{2 \pi a}{P} \sin (i) \frac{M_{\mathrm{p}}}{M_{\mathrm{p}}+M_{\mathrm{s}}} \sin (\phi)$, where a is the semi-major axis, $P$ is the orbital period, $i$ is the orbital inclination, and $\phi$ is the orbital phase. The shaded region was calculated by error propagation of the values in the previous expression. The lower panel shows the residuals for the fit.

Therefore, if the scientific program requires high-precision radial velocity measurements, we strongly recommend to obtain arc calibrations before and after each science image.

As was said in previous sections, during the commissioning run we observed some already known planets to test the CAFE capabilities with real data. Spectra of parent stars TrEs-3 described in O'Donovan et al. (2007) and TrEs-2 described in O'Donovan et al. (2006) were acquired in different phases of their orbits.

The first system is composed of an $M_{\mathrm{p}} \sin (i)=1.91_{-0.08}^{+0.06} M_{\mathrm{J}}$ planet orbiting a G-type star (TrES-3) of $M_{\mathrm{s}}=0.924_{0.04}^{+0.012} M_{\odot}$. The physical and dynamical characteristics of this system causes a movement of the parent star around the center of masses with an amplitude of $K=371 \pm 41 \mathrm{~m} / \mathrm{s}$ (error calculated during the orbit's quadrature). In Fig. 16, we show the observational points taken with CAFE after reducing the raw data with the pipeline explained in the previous section. The radial velocity for each spectrum was derived by cross-correlating the 50 orders with the highest $\mathrm{S} / \mathrm{N}$ with a solar spectrum ${ }^{5}$. When combining all

\footnotetext{
5 http://wWw. bass2000. obspm. fr
}

cross-correlation functions of the orders, we weigthed them according to their FWHM. We also plot in Fig. 16, the radial velocity curve according to the published parameters of the system $^{6}$. We obtain a nice fit whose highest residuals are of the order of $50 \mathrm{~m} / \mathrm{s}$.

We proceeded in the same manner for the second star, TrES-2, hosts a planet lighter than TrES-3b, which is less massive than $\operatorname{TrES}-3 \mathrm{~b}\left(M_{\mathrm{p}} \sin (i)=1.253 \pm 0.052 M_{\mathrm{J}}\right)$ and orbits a heavier star of $M_{\mathrm{S}}=0.980 \pm 0.062 M_{\odot}$. Hence the amplitude of the radial velocity curve is significantly flatter, $K=190 \pm 15 \mathrm{~m} / \mathrm{s}$. Although, in this case, a somewhat poor sampling of this curve was taken, we can see in Fig. 11 that lower residuals were obtained. Again, observed points with CAFE fit what is expected for this system, with residuals lower than $20 \mathrm{~m} / \mathrm{s}$.

According to our observations of radial velocity standards, we find precisions of $22 \mathrm{~m} / \mathrm{s}$ for HD 124292 when we remove the night-by-night trend (see Fig. 17). This trend could be caused by different effects such as stellar pulsation or night-by-night instrumental effects due to some small problems releated to the arc

6 Obtained from the http://www. exoplanet. eu website 
J. Aceituno et al.: CAFE: Calar Alto Fiber-fed Échelle spectrograph

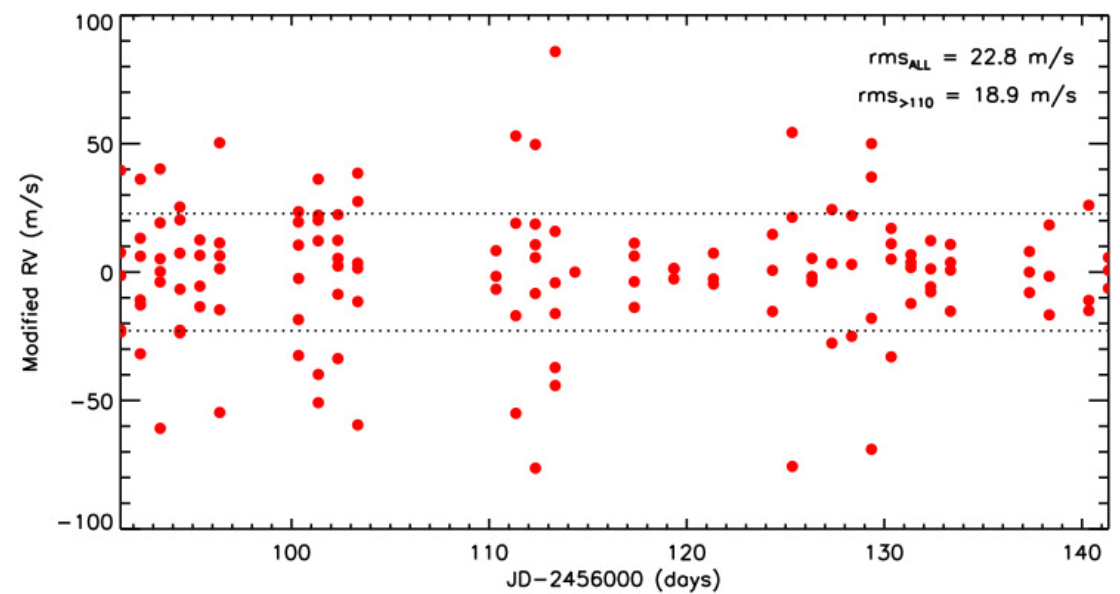

Fig. 17. rms error of radial velocity measurements of the standard HD 124292. lamp intensity stability. This is basically because a certain temperature is required for the lamp to produce sharp emission lines, and not broader ones, whose centroids are different. Only when the lamp is switched on during the complete nights the best stability in the position of the arc-lamps is reached. However, this is not possible because there may be effects in straylight.

These radial velocity measurements were determined with high $\mathrm{S} / \mathrm{N}$ spectra (greater than 80 ) of bright known standards and by cross-correlating these with synthetic spectra of the same spectral type. The higher rms obtained for TrEs-3b could be due among other things to the fact that the Tres- 3 spectra have a lower S/N (around 30).

\section{Summary and conclusions}

We have presented the design, manufacturing, and performance analysis of CAFE. We showed that the instrument was built according to demanding requirements, which resulted in an instrument of excellent stability and efficiency. First tests at the telescope were presented and led to very encouraging results, which can be summarized as follows:

- The instrument is fully operational and publically accessible.

- The resolution estimated on real data corresponds to $62000 \pm 5000 \mathrm{~A}$

- Based on real observations, the limiting magnitude of CAFE is $\sim 15 \mathrm{mag}$ for an exposure time of one hour, with an $\mathrm{S} / \mathrm{N}$ of $\sim 20$.

- Two known planets were observed and their radial velocities measured to test the CAFE capabilities.

- The high stability of the instrument shows that a radial velocity precision of about $\sim 20 \mathrm{~m} / \mathrm{s}$ can be achieved.
Acknowledgements. The authors thank the sub-programs of Viabilidad, Diseño, Acceso y Mejora de ICTS , ICTS-2008-24 and ICTS-2009-32, the PAI Proyecto de Excelencia P08-FWM-04319 and the funds of the PAI research group FQM360, and the MICINN program AYA2010-21161-C02-02, AYA201022111-C03-03, CDS2006-00070 and PRICIT-S2009/ESP-1496. We are grateful to the Calar Alto staff and in particular to E. de Guindos and E. de Juan for the computer support, to S. Reinhart, M. Pineda, A. Garcia, and J. Garcia, who partially managed the mechanical assembling; to N. Vico, L. Hernandez, J. F. Lopez, and J. Marin, for their contributions to the new electronic controller and to V. Gomez, M. Aguila, and R. Lopez who prepared the room that holds the instrument.We also thank to the referee, whose comments have improved this manuscript.

\section{References}

Avila, G. 1988, in Fiber Optics in Astronomy, ed. S. C. Barden, ASP Conf. Ser., 3,63

Avila, G., Singh, P., \& Albertsen, M. 2006, in SPIE Conf. Ser., 6269

Crause, L., Bershady, M., \& Buckley, D. 2008, in SPIE Conf. Ser., 7014

Guenther, E., Stecklum, B., \& Klose, S. 1999, Optical and Infrared Spectroscopy of Circumstellar Matter, ASP Conf. Ser., 188

Kaufer, A., Wolf, B., Andersen, J., \& Pasquini, L. 1997, The Messenger, 89, 1

Kaufer, A., Stahl, O., Tubbesing, S., et al. 1999, The Messenger, 95, 8

O’Donovan, F. T., Charbonneau, D., Mandushev, G., et al. 2006, ApJ, 651, L61

O’Donovan, F. T., Charbonneau, D., Bakos, G. Á., et al. 2007, ApJ, 663, L37

Pepe, F., Mayor, M., Delabre, B., et al. 2000, in SPIE Conf. Ser. 4008, eds. M. Iye, \& A. F. Moorwood, 582

Perryman, M. 2003, in GQAIA Spectroscopy: Science and Technology, ed. U. Munari, ASP Conf. Ser., 298, 3

Pfeiffer, M. J., Frank, C., Baumueller, D., Fuhrmann, K., \& Gehren, T. 1998, A\&AS, 130, 381

Pronik, V. I. 1995, Izvestiya Ordena Trudovogo Krasnogo Znameni Krymskoj Astrofizicheskoj Observatorii, 89, 111

Sánchez, S. F. 2006, Astron. Nachr., 327, 850

Sánchez, S. F., Aceituno, J., Thiele, U., Pérez-Ramírez, D., \& Alves, J. 2007, PASP, 119, 1186

Sánchez, S. F., Kennicutt, R. C., Gil de Paz, A., et al. 2012, A\&A, 538, A8 